\title{
SMOKING-RELATED DNA ADDUCTS AS POTENTIAL DIAGNOSTIC MARKERS OF LUNG CANCER: NEW PERSPECTIVES
}

\author{
E.S. Grigoryeva ${ }^{1,2 *}$ D.A.Kokova', A.N.Gratchev ${ }^{1}$, E.S. Cherdyntsev $^{3}$, M.A. Buldakov ${ }^{2,3}$, \\ J.G. Kzhyshkowska, ${ }^{1,4}$ N.V.Cherdyntseva, ${ }^{1,2}$ \\ ${ }^{1}$ National Research Tomsk State University, Tomsk 634050, Russia \\ ${ }^{2}$ Tomsk Cancer Research Institute, Tomsk 634050, Russia \\ ${ }^{3}$ National Research Tomsk Polytechnic University, Tomsk 634050, Russia \\ ${ }^{4}$ Department of Innate Immunity and Tolerance, Institute of Transfusion Medicine and Immunology, \\ Medical Faculty Mannheim, University of Heidelberg, Mannheim D-68167, Germany
}

\begin{abstract}
In recent years, the new direction such as identification of informative circulating markers reflecting molecular genetic changes in the DNA of tumor cells was actively developed. Smoking-related DNA adducts are very promising research area, since they indicate high pathogenetic importance in the lung carcinogenesis and can be identified in biological samples with high accuracy and reliability using highly sensitive mass spectrometry methods (TOF/TOF, TOF/MS, MS/MS). The appearance of DNA adducts in blood or tissues is the result of the interaction of carcinogenic factors, such as tobacco constituents, and the body reaction which is determined by individual characteristics of metabolic and repair systems. So, DNA adducts may be considered as a cumulative mirror of heterogeneous response of different individuals to smoking carcinogens, which finally could determine the risk for lung cancer. This review is devoted to analysis of the role of DNA adducts in lung carcinogenesis in order to demonstrate their usefulness as cancer associated markers. Currently, there are some serious limitations impeding the widespread use of DNA adducts as cancer biomarkers, due to failure of standardization of mass spectrometry analysis in order to correctly measure the adduct level in each individual. However, it is known that all DNA adducts are immunogenic, their accumulation over some threshold concentration leads to the appearance of long-living autoantibodies. Thus, detection of an informative pattern of autoantibodies against DNA adducts using innovative multiplex ELISA immunoassay may be a promising approach to find lung cancer at an early stage in high-risk groups (smokers, manufacturing workers, urban dwellers).
\end{abstract}

Key Words: lung cancer, DNA adducts, tobacco smoking.

\section{INTRODUCTION}

Cancer is one of the leading causes of death in the world, accounting for over $25 \%$ of all deaths in developed countries. Lung cancer ( $\mathrm{LC}$ ) holds the top position in cancer morbidity and mortality among men worldwide. The both sexes combined, LC incidence rate was 23.1 cases/100 000 in the world and $28.7 / 100000$ cases in Central and Eastern European countries. The LC mortality worldwide was 19.7 cases/100000 vs 24.1 cases/100000 in Europe [1]. At the same time, the mortality during the first year after diagnosis is very high and is about $67 \%$. Success of surgical treatment is closely related to the opportunity for early diagnosis of LC. The importance of early diagnosis is confirmed by the 5 -year survival rate after radical surgery. People diagnosed with stage I and II of LC tend to have higher 5-year survival rate (63.5\%, and $43.5 \%$, respectively) than people diagnosed with stage III $(22.9 \%)[1,2]$.

Computed tomography is usually used for diagnosis and screening of LC, but this method has several disadvantages such as a high cost and low availability as well as a lack of sensitivity and specificity. Diagnostic errors occur in $25 \%$ of cases resulting in treatment delay of up to one year. Usage of new diagnostic methods,

Submitted: December 04, 2014.

*Correspondence: E-mail: grigorieva@oncology.tomsk.ru Abbreviations used: BPDE - benzopyrene-7,8-diol-9,10-epoxide; LC - lung cancer; PAHs - polycyclic aromatic hydrocarbons; PET - positron emission tomography. i.e. positron emission tomography (PET) and autofluorescence bronchoscopy for the detection of LC has not reduced the mortality [3] due to the low efficiency of these methods at early stages of the disease.

The usage of circulating tumor-associated molecular markers in combination with the methods of instrumental imaging is one of the most promising trends for improving the effectiveness of early diagnosis of LC [4]. Specific molecular markers can also be used for differential diagnosis of LC and other pathological processes in lung, for detection of drug resistance formation, to adapt treatment accordingly depending on what the chemotherapy drug can be changed [5]. There are several circulating protein markers which are practically used in diagnostics and monitoring of LC, namely, CEA (carcino-embrionic antigen), CYFRA-21-1 (CYtokeratin FRAgment), TPA (tissue plasminogen activator), Pro-GRP (pro-gastrin-releasing peptide), NSE (neuron-specific enolase). However, current serological markers of LC have very low sensitivity. For example, CEA, NSE, CYFRA-21-1 measurement can detect non-small cell LC with a sensitivity of 55; 38 and $65 \%$ and specificity of $70 ; 97$ and $87 \%$, respectively [6].

In recent years, the new direction such as identification of informative circulating markers reflecting molecular genetic changes in the DNA of tumor cells is actively developed. There is a large number of reviews highlighting the possibility of using circulating tumor DNA for the diagnosis and prediction of clinical course of cancer [7-9].

These markers include tumor-specific mutations, chromosomal aberration, methylated DNA fragments, 
and DNA adducts caused by tobacco smoke carcinogens as a main etiologic factor of LC.

DNA adducts are very promising research area, since their chemical structures are considerably different from mutated or methylated DNA and can be identified in clinical samples with high accuracy and reliability. Currently, there are highly sensitive mass spectrometry methods (TOF/TOF, TOF/MS, MS/MS), to identify and quantify smoking-related DNA adducts [10]. Continuously updated information about the genotoxic effect of tobacco smoke components indicates their high pathogenetic importance in the tumor formation.

This review is devoted to the analysis of the role of DNA adducts in lung carcinogenesis in order to demonstrate their usefulness as noninvasive cancer associated markers. The perspective to use them as cancer markers is provided by their crucial role in smoking-related lung carcinogenesis. The further investigations using mass spectrometry analysis are needed to identify the most important DNA adducts reflecting the risk of cancer development. DNA adducts are known to be high immunogenic substances resulting in the autoantibody formation in the blood. The testing of autoantibodies against reliable DNA adducts may be considered as a promising way for early detection of LC, however further studies are required to demonstrate clinical validity and utility for this approach.

\section{THE ROLE OF TOBACCO SMOKING IN THE PATHOGENESIS OF LUNG CANCER}

Complex composition of tobacco smoke makes difficult to identify the mechanisms that trigger the process of carcinogenesis. Approximately 20 substances in cigarette smoke have a carcinogenic effect, which are represented by polycyclic aromatic hydrocarbons (PAHs), nitrosamines, aldehydes, etc. [11]. All of them are on the list of carcinogens released by IARC (International Agency for Research on Cancer) [12]. In this review, we made an attempt to summarize the mechanisms of carcinogenic action of tobacco smoke components that can cause genetic alterations in oncogenes and tumor suppressor genes, as well as to show the possible causes of the heterogeneity of the negative effects of smoking in different subjects.

Nicotine, compound which is found in large quantities in tobacco, has no carcinogenic effect, but it is a highly addictive substance that leads to a constant consumption and accumulation of other carcinogens of tobacco smoke in the body. Some of the major components of tobacco smoke such as PAHs and nitrosamines are not carcinogens initially they need metabolic activation to acquire carcinogenic properties. Imbalance between metabolic activation and detoxification may lead to an increased risk of LC [13]. Normally, the cell with damaged DNA commits suicide by apoptosis, but if these mutations occur continuously in the critical region of an oncogene, this may lead to activation and accumulation of aberrant cells with a loss of cell cycle regulation, and as a result to malignant transformation.

Molecular genetic changes in LC inducing by tobacco smoking are represented by the following events: chro- mosomal aberrations, point mutations, dysregulation of oncogenic signal transduction pathways, DNA methylation, etc. Active or passive exposure to tobacco smoking equally causes multiple cytogenetic changes, including different types of DNA and chromosome damage. Point mutations in oncogenes and tumor suppressor genes are quite frequently detected in the tumor tissue of $\mathrm{LC}$ patients who are former smokers. According to published data, TP53 mutations are associated with exposure to tobacco smoke and may cause disruption of its functional activity.

The increased frequency of nucleotide substitution of thymine to guanine in TP53 gene in LC smokers often observed in the so-called "hot spots" which are located at $157,158,245,248$ and 273 codons [14-16]. It has been experimentally proved that the appearance of such nucleotide substitution may be caused by the action of tobacco smoke metabolite benzo[a]pyrene - benzopyrene-7,8diol-9, 10-epoxide (BPDE) leading to formation DNA adducts in these regions $[16,17]$.

Methylation of several suppressor genes, namely, CDKN2A (cyclin-dependent kinase inhibitor 2A), DAPK1 (death-associated protein kinase 1), RASSF1A (Ras association domain family 1 isoform), RARb2 (retinoic acid receptor B2), APC (adenomatous polyposis coli), $\mathrm{CDH} 13$ (cadherin 13), MGMT (O(6)-methylguanineDNA methyltransferase), MLH1 (MutL homolog 1), MSH2 (MutS protein homolog 2) and GSTP1 (glutation$S$-transferase $P$ 1) resulting in their inactivation has been found in LC [18]. Number of studies demonstrate that methylation of the CDKN2A gene is much less common in non-smoking patients with LC, indicating a direct link with smoking. Also, a lower rate of the APC gene methylation $(p<0.0001)$ was found in patients who had never smoked. Methylated forms of these genes are detected in DNA from sputum, bronchoalveolar lavage fluid and serum, suggesting the possibility of their use for minimally invasive screening. The possible significance of these markers as risk factors for $L C$ is shown in examples of RARB2 and CDKN2A genes, their methylated forms are detected in bronchial biopsy samples of smokers without tumor and serum of patients with an increased risk of $L C$, and in serum of squamous cell lung carcinoma patients 3 years before the clinical manifestation $[19,20]$. Prognostic role of methylated DNA fragments of RASSF1A and RARb2 suppressor genes in serum patients with squamous $L C$ was shown in relation to risk of relapse after combined treatment [21, 22].

The active components of tobacco smoke affect basic cellular functions, such as cell response to DNA damage, induction of oxidative stress, apoptosis and inflammatory reactions. Taking into account the involvement in these processes a large number of functionally important genes and signaling pathways necessary is to clarify the initial changes caused by metabolites of tobacco smoke that can further lead to tumor formation.

Oxidative stress is a result of an imbalance between the formation of reactive oxygen species (ROS) and a biological system's ability to readily detoxify the reactive intermediates or to repair the resulting damage. Oxidative stress is an important phenomenon in the pathophysio- 
logy of cancer [23]. Oxidative stress induced by the tobacco smoke components leads to DNA strand breaks and oxidative modification of DNA. Tobacco smoke contains a large number of free radicals which cause DNA damage and generate oxidized base forms such as 7,8-dihydro-8-oxo-2-deoxyguanosine (8-OH-dG), which is a classical biomarker of oxidative DNA damage. Inflammatory response induced by damaging effect of tobacco smoke creates certain conditions in microenvironment that enhances carcinogenesis [24]. These conditions include glutathione oxidation, followed by increasing of glutathione disulfide level in the lung tissue and bronchoalveolar lavage fluid; increase in 8-OH-DG, overexpression of mRNA of nitric oxide synthase and endothelial nitric oxide synthase genes in the lung tissue; reduction of antioxidants in the blood, such as methylumbelliferone glucuronide and ferroxidase; increasing lipid peroxidation indicators (8-Epi-prostaglandin F2 alpha); increasing oxygen removing from leukocytes in bronchoalveolar lavage fluid [25]. All these processes contribute to the accumulation of genotoxic damage and the development of chronic inflammatory response aimed at preserving tissue integration, triggering processes of repair and regeneration, similar to wound healing. In this case, the inflammatory response performs the tumor-promoting function by production of growth, anti-apoptotic, pro-angiogenic and matrix remodeling factors [26].

Recent studies have shown that mutations or aberrant expression of miRNAs that are negative post-transcriptional regulators of gene expression associated with various human diseases, including cancer. Various researches on non-small cell LC samples established the potential association between the expression of different miRNAs and action components of tobacco smoke. For example, expression of miR-218 significantly reduced in the tumors of patients - current or former smokers, while in tumors of patients who has never smoked such changes were not detected. Another study showed a significant difference in the expression levels of miR-130a between smoking and nonsmoking patients with LC [27]. In a recent study it was found that the expression level of miR-143 was lower in smokers than non-smokers patients, as the authors suggest, miR-143 may play a significant role in the etiology of LC [28]. In contrast to the above, microarray study performed by Landi et al. did not reveal any significant differences in the miRNAs expression in smokers and non-smoking patients with LC [29]. Inconsistency of the results can be explained by a small number of the samples. Probably more detailed study with strict stratification of patients according to histological type of tumor, smoking status, gender is required.

Still it has not been reliably established, whether tobacco smoke-induced LC characterized by special molecular signature or all of the molecular changes in LC are universal. Detection of specific genetic and epigenetic abnormalities induced by tobacco smoke will allow identifying the key genes associated with potentially high risk for LC. This will help to carry out monitoring of patients with pre-cancerous conditions of the lung for the early detection of the disease and to plan strategies for prevention.

\section{CAUSES OF HETEROGENEITY IN THE NEGATIVE EFFECTS OF SMOKING}

The role of smoking in $L C$ development was finally proved only in the 60s of the last century, because it was unclear why incidence of LC in smokers was extremely low and amounted to only $10 \%$. The subsequent development of molecular biological research methods allowed comes closer to the cause of such ambiguity. Apparently, genetically determined (or inherited) characteristics of metabolic system, as well as DNA repair system, make a significant contribution to the risk of LC. In particular tobacco smoke carcinogens undergo complex metabolic conversions, usually via series of chemical reactions. It is known that people who inherited different polymorphic variants of genes encoding enzymes involved in carcinogen metabolism characterized by different degrees of resistance to their action and, accordingly, various risk of LC development [30-32].

After entering the body PAH molecules induce the expression of Phase I and Phase II metabolic enzymes [33], including aldo-keto reductases, cytochrome P450, catechol-O-methyltransferase, epoxide hydrolases, peroxidases, glutathione S-transferase (GSTM1), Nacetyltransferases, sulfotransferases, and other enzymes which catalyze the conjugation reaction [34]. The most common mechanism of PAH metabolic activation, for example, benzo[a]pyrene, is the formation of the dihydrodiol epoxide - BPDE, which is catalyzed by cytochrome P450 enzymes and epoxide. The main enzymes which involved in PAHs metabolism are Cytochromes P450 CYP 1A1, CYP 1A2, CYP 1B1 and CYP 3A4. Induction of these enzymes is significantly increased in the presence of benzo[a]pyrene and some polyhalogenated hydrocarbons. People who have inherited low-activity variants of cytochrome are relatively resistant to tobacco smoke carcinogens. In particular, it has been found that some polymorphic variants of CYP $1 \mathrm{~A}$ gene associated with increased formation of DNA adducts and mutagenesis in some populations [35].

Subsequent phase of PAH metabolism includes conjugation of phase I metabolites with small molecules. Enzymes catalyzing phase II include sulfotransferase, UDP (Uridine 5'-diphospho)-glucuronyl transferase and glutathione-S-transferase. Sulfotransferases (SULT) involved in the activation of such PAH metabolites as 7, 12-dimethylbenz(a)anthracene and its methylhydroxylated derivatives in various tissues. It is shown that polymorphic variants of sulfotransferase SULT1A1 are associated with different levels of DNA adducts formation [36]. Conjugation of PAH metabolites with glucuronic acid (glucuronidation) is also one of the main way of detoxification resulting in formation of polar conjugates, which have higher excretion rate. Oxidized derivatives of benzo[a]pyrene (for example, 1-hydroxypyridine) are a substrate for UDP-glucuronosyltransferase. The resulting compound 1-hydroxypyrene glucuronide can be used as biomarker of PAH exposure [37]. Glutathione- 
S-transferase is also involved in the conjugation processes of PAH derivatives. Study conducted on placental tissue samples showed that the activity of glutathione-Stransferase determined by glutathione redox status and the presence of genotoxic damage that may be used as a marker for the damaging effects of PAHs [38].

Polymorphisms in phase II metabolic enzymes genes are correlated with the amount of DNA damageand, therefore, with a risk of malignization. Thus, Binkova et al. [39] demonstrated an association between the polymorphic variants of a glutathione-S-transferase and the amount of DNA adducts. Individuals who have functionally inactivated glutathione transferase $\mu$ gene characterized by increased susceptibility to LC. It is not surprising that the most dangerous is combination of unfavorable genotypes of CYP $1 \mathrm{~A} 1$ and GSTM1; individuals with such combination have 2-fold increased risk of LC development [40, 41].

The contribution of the genetic component in the formation of LC risk is not limited to only the genes responsible for the metabolic activation of tobacco smoke components. A significant role is also played by the individual characteristics of the DNA repair system. The most common mechanisms of cell repair include: nucleotide excision repair (NER), base excision repair (BER), nonhomologous end joining (NHEJ), homologous recombination repair (HRR), transcription coupled repair (TCR). Several studies have shown that polymorphisms of certain genes of NER increase the efficiency of repair [42].

Four known polymorphism - 4G/A substitution in XPA (xeroderma pigmentosum, complementation group $\mathrm{A}$ ) gene, C8092A in ERCC1 (excision repair cross-complementation group 1) gene, Lys751GIn in XPD (xeroderma pigmentosum group D, ERCC2) gene and Ser835Ser in XPF (xeroderma pigmentosum group F, ERCC4) gene are associated with reduced DNA repair capacity and increased incidence of tumors of various types [43].

Recentstudies have shownthatBER playsa special role in the repair of damage produced by the action of PAHs. It was found that the $\mathrm{CHO}$ (Chinese hamster ovary) cell line deficient in BER genes more sensitive to 11,12-dihydroxy-13,14-epoxy-11,12,13,14-tetrahydrodibenzo[a,I] pyrene (DBPDE), resulting in an increase in the frequency of chromosome aberrations [44]. Polymorphic variants of BER genes are associated with an increased incidence of DNA damage. Thus, the product of theXRCC1 (X-rayrepair cross-complementing protein 1) gene has 4 functional polymorphic variants - T77C, Arg194Trp, Arg280His and Arg399GIn [45], which are associated with altered ability to repair DNA damage caused by PAH action [46].

Polymorphisms of proteins involved in HRR, is probably able to protect the effects of PAHs. One of these proteins, XRCC3, is involved in homologous recombination repair of double strand breaks and removing of double bonds (cross-linking) between guanine bases belonging to different chains. According to Shen et al. (2003), polymorphism of XRCC3 codon 241 is protective for bladder cancer, especially for long-term smokers [47]. Considering that tobacco smoke contains a large number of PAH, this fact allows making sure that HRR involved in repair of DNA lesions induced by exposure to PAHs. Furthermore, it has been shown in vitro that $\mathrm{CHO}$ cell line deficient in $\mathrm{HRgenes}$ are more susceptible to the adverse effects of PAHs, which also confirms the data of the important role of homologous recombination in DNA repair [44].

\section{DNA ADDUCTS AS MARKERS OF LUNG CANCER RISK}

Polycyclic aromatic hydrocarbons. Initially PAHs are absolutely inert substances and acquire their biological activity only after metabolic activation. Among PAHs, benzo[a]pyrene is the well-studied compound and its ability to induce lung tumors when administered topically and inhaled well described [48].

Benzo[a]pyrene forms at least nine different DNA adducts which have been characterized and isolated from body fluids (saliva, urine, blood), lung, liver and cells of human and animals. Different types of adducts were determined by mass spectrometry and were shown specific sites of DNA damage. There are three metabolic pathways of benzo[a]pyrene activation formation of a radical cation, diol epoxide and orthoquinone [49]. The third pathway leads to the formation of reactive metabolite - benzo[a]pyrene-7,8-dione by oxidation of benzo[a]pyrene-7,8-trans-dihydrodiol catalyzed by aldo-keto reductases. Thus, itwas shown that H358 cells produce large amount of benzopyrene-7,8dione under exposure to benzo[a]pyrene. In subsequent experiments it was found that 7,8-benzopyrene-dione DNA-adducts detected in immortalized HBEC-KT cells and in human lung adenocarcinoma cells A549. Wherein A549 cells mainly produce adducts of 2'-deoxyguanosine (dGuo), while HBEC-KT cell lines - 2'-deoxyadenosine (dAde). Mass spectrometric study allowed to identify several stable benzo[a]pyrene-7,8-dione adducts: hydrated forms of benzo[a]pyrene 7,8-dione-N2-dGuo and benzo[a]pyrene-7,8-dione-N1-dGuo in A549 cells; hydrated and unhydrated forms of benzo[a]pyrene-7,8dione-N1-dAde and benzo[a]pyrene-7,8-dione-N3-dAde in HBEC-KT cells.

Another product of benzo[a]pyrene metabolic activation is benzopyrene-7,8-diol-9,10-epoxide. All diol epoxides exist as diasteromers, i.e. stereoisomers that are not mirror images of each other, and referred to as antiBPDE and syn-BPDE, each of which at one time may be represented by two (+/-) enantiomers. The experiment conducted by Buening et al. showed that isomer $(+)-$ anti-BPDE has the greatest tumorigenicity in vivo [50]. It is known that indicated isomer interacts with N2-atom of deoxyguanosine forming (+)-trans, (+)-cis-BPDE-N2$\mathrm{dG}$, and (-)-trans and (-)-cis-BPDE-N2-dG adducts. Of these four adducts (+)-cis-BPDE- N2-dG is the most common and its presence is registered in $45 \%$ of smokers. Presence of BPDE-DNA adducts in human tissues has been reliably established [51] and shown to be the highest concentration in the bronchial epithelium, indicating a direct role in the initiation of LC [52].

Aromatic amines. Aromatic amines contained in the tobacco smoke can also be sources for DNA adduct formation. The most studied aromatic amine in tobacco smoke is a 4-aminobiphenyl (4-ABP) [53]. A recent study 
authored by Lee et al. found that 4-ABP-adducts are formed mainly in the mutational hot spots of TP53 gene (codons 280 and 285) in a human bladder cancer cell line [53]. These results confirm pathogenic role of 4-ABPadducts in the development of bladder cancer. Using of sensitive immunobiological and mass spectrometric methods allows detecting the presence of DNA and protein 4-ABP-adducts in various human tissues resulting from exposure to tobacco smoke.

It is interesting that in intact breast tissue adjacent to the tumor, level of 4-ABP-adducts correlated with smoking status. In biopsies obtained from patients with bladder cancer quantity of DNA adducts were also associated with smoking and the tumor size. However, the results obtained in studies of different groups of patients are rather contradictory. Thus, using of HPLC (high-performance liquid chromatography) method, followed by tandem mass spectrometry does not allowed the identification of 4-ABP-adducts in biopsies of 70 breast cancer patients. Perhaps this may be due to incorrect grouping of patients, as a systematic survey and detection of patient's smoking status has not been established. The few studies of aromatic amines DNA adducts in the tissues of smokers and non-smokers LC patients are also characterized as a highly controversial [54].

Aldehydes. Acrolein (2-propenal) - highly reactive unsaturated aldehyde contained in thermally processed foods and in the environment, as the product of incomplete combustion of gasoline, plastic and wood. Tobacco smoke contains approximately $180 \mathrm{mg}$ of acrolein per cigarette, which is 1000 times greater than the PAHs content. In this case, unlike PAHs, acrolein is initially active component capable of providing a damaging effect. Acrolein has a mutagenic effect toward bacterial and mammalian cells. Acrolein binds deoxyguanosine forming one of two stereoisomers, 1,N2-propanodeoxyguanosine (Acr-dGuo) - a-OH-Acr-dGuo and $\gamma-\mathrm{OH}$-Acr-dGuo. Highest mutagenic effect has $\alpha$-isomer which induce G>T substitutions in CpG-islands, mainly, in TP53 codons 249 and 273 [55].

One of the first studies authored by Nath et al. (1998) demonstrated a significant increase of Acr-dGuo adducts in epithelial cells of the oral mucosa in smokers [56]. Based on the similarity of the mechanisms of acrolein and PAHs action, many authors have suggested that indicated major component of tobacco smoke may be responsible for the TP53 gene mutations in lung cancer associated with smoking. To detect the presence of acrolein adducts in the lung tissue and to evaluate their contribution in the pathogenesis of LC, Zhang et al. developed a methodological approach based on liquid chromatography and tandem mass spectrometry to quantify Acr-dGuo in biological samples [57]. They analyzed 30 samples of normal lung tissue and detected the presence of both stereoisomers in all samples irrespective of smoking status. In the next study, the presence of Acr-dGuo adducts was evaluated in peripheral blood lymphocytes of 25 smokers and 25 non-smokers. Predominant presence $\gamma$-isomer was detected in all samples except three patients. However, significant differences between smokers and non-smokers were not found. Paradoxically, the average content of $\mathrm{Y}-\mathrm{OH}-\mathrm{Acr}$-dGuo was statistically higher in nonsmokers. Based on these results, the authors conclude that acrolein probably undergoes detoxification with a very high efficiency by conjugation with glutathione. Obviously, the most damaging effect of acrolein adducts, promoting malignant transformation of epithelial cells of the lung, is the inhibition of NER, which is responsible for the removal of DNA damage caused by the action of PAHs [55].

Acetaldehyde is the one of the most common carcinogens in tobacco smoke, may also be present in foods and in products of combustion. A certain amount of acetaldehyde is formed endogenously in threonine catabolism and metabolism of ethanol. Acetaldehyde binds DNA forming N2-ethylidene-dGuo. After enzymatic hydrolysis of DNA in the presence of $\mathrm{NaBH} 3 \mathrm{CN}$ indicated metabolite forms N2-ethyl-dGuo, which can be detect by mass spectrometry [58]. Theoretically, N2-ethylidene-dGuo may undergo a subsequent transformation forming 1,N2propanodeoxyguanosine (the same adduct formation occurs under the exposure of acrolein). The proof of this hypothesis serves an experiment in vivo conducted byWang etal. (2006) [58]. 1,N2-propanodeoxyguanosine can be detected in the cells after exposure to micromolar concentrations of acetaldehyde. Probably acetaldehyde adducts play a role in the carcinogenic effect of tobacco smoke, but the contribution of this component to the overall genotoxicity should be clarified in further studies.

The evidence exists that, among other components there are some ethylating agents in a tobacco smoke whose structure has not yet been established [59]. When studying normal lung epithelium obtained from patients with LC, O4-etiltimidin was detected in 10 of 13 smokers and only 3 out of 11 non-smoking subjects. Another study on a small group of patients demonstrated the presence of the O4-ethyltimidine in sputum from two of four smokers and was not detected in either of the samples obtained from nonsmokers [60].

Nitrosamines. Significant contribution to the study of nitrosamines metabolism contained in tobacco smoke was made by Hecht and co-authors [61]. For metabolite identification the authors used the HPLC-electrospray ionization-MS-MS analysis in vitro and in vivo studies. It was found that these chemicals act as bidentate (ligand that binds through two sites) carcinogens. Contained in cigarette smoke nitrosamine 4-(methylnitrosamino)1-(3-pyridyl)-1-butanone (NNK) undergoes metabolic activation, forming 4-(methylnitrosamino)-1-(3-pyridyl)1-butanol (NNAL). The further reaction consists of conversion of intermediate metabolite to methanediazohydroxid, which in its turn interacts with DNA and forms O6-methyl-dGuo, 7-methyl-dGuo and O6-methyl-dThd. O6-methyl-dGuo has the highest mutagenic effect [62]. In addition to the well-studied pathway of activation, leading to the formation of a methylating agent, there is another mechanism which results in the formation of pyridyloxobutylating agent. Another nitrosamine $\mathrm{N}$-nitrosonornicotine (NNN) also produces two types of reactive agents. Both compounds have a pronounced carcinogenic effect in animal models, forming tumors with 
similar localization to smokers with LC [63]. Pathway which associated with pyridyloxobutylation leads to the formation of a large number of adducts: 7-[4-(3-pyridyl)-4-oxobut1-yl]-2'-deoxyguanosine (7-pobdG), O2-[4-(3-pyridyl)4-oxobut-1-yl]-2'-deoxycytosine(O2-pobdC), O2-[4-(3pyridyl)-4-oxobut-1-yl]-2'-deoxythymidine(O2-pobdT), O6-[4-(3-Pyridyl)-4-Oxobut-1-yl]-2'-Dexoyguanosine (O6-pobdG). Two of these adducts - 7-pobdG and O2pobdC release appropriate nitrogenous base, forming 7-[4-(3-pyridyl)-4-oxobut-1-yl]-guanine (7-pobG) and O2-[4-(3-pyridyl)-4-oxobut-1-yl]-cytosine (O2pobC). The presence of all these adducts was detected in the tissues of rodents after administration of NNN and NNK. Moreover, the maximum concentration was detected in lung, liver and then, in mucosa of the nasal cavity and pancreas. If one considers the ratio of different adducts their distribution in the lung tissue, was as follows: O2-pobdT $\geqslant 7$-pobG $>02-$ pobC $>>06-$ pobdG.

Interestingly, the study of the content of these adducts in tissues of patients with LC have not been conducted. These data could clarify the role of nitrosamines in the pathogenesis of LCassociated with smoking. Study of Bessette et al. [64] demonstrated that $\mathrm{N}$-(deoxyguanosine8-yl)-2-amino-1-methyl-6-phenylimidazo[4,5-b] pyridine was detected in saliva samples of 13 out of 29 smokers and only in 2 out of 8 samples of nonsmokers, while a $\mathrm{N}$-(deoxyguanosin-8-yl)-2-amino-9Hpyrido[2,3-b]indole and $\mathrm{N}$-(deoxyguanosin-8-yl)2-amino-3,8-dimethylimidazo[4,5-f]quinoxaline were detected in saliva samples only in three smokers and $\mathrm{N}$-(deoxyguanosin-8-yl)-4-4-aminobiphenyl was detected in saliva of two smokers. The levels of these various adducts ranged from 1 to 9 adducts per 108 base pairs.

Formaldehyde. Recent mass-spectrometric studies comparing the level of formaldehyde DNA adduct in smokers and non-smokers showed that the level of N6-hydroxymethyldeoxyadenosine (N6-HOMe-dAdo) in smokers was higher than in non-smokers [65], that may indicate a role of formaldehyde in the pathogenesis of LC induced by smoking.

Formaldehyde is also a component of tobacco smoke, which is classified as carcinogenic and genotoxic agent, forming DNA adducts. Meta-analysis conducted by US researchers of more than 25 thousand manufacturing workers who are exposed to formaldehyde, showed a significant increase in the risk of leukemia. In the study held by Wang et al. quantity of N6-HOME-dAdo was measured using mass spectrometry in leukocyte DNA from 32 smokers and 30 non-smokers [65]. The presence of this adduct was detected in 21 out of 29 smokers (179 \pm $205 \mathrm{fmol} / \mu \mathrm{mol} d \mathrm{ddo}$ ) and only in 7 out of 30 non-smokers $(15.5 \pm 33.8 \mathrm{fmol} / \mu \mathrm{mol} \mathrm{dAdo})$. However, the study of Lu et al. [66] showed that formaldehyde metabolites form DNA adducts, preferably binding to deoxyguanosine and forms unstable N2-hydroxymethyl-dGuo, which is converted in N2 -methyl-dGuo in the presence of sodium cyanoborohydride. At the same time, the presence of formaldehyde adducts detected mainly in the mucosa of the nasal cavity, lung tissue, but not in distant organs such as liver, spleen, bone marrow.
Estimation of the contribution of formaldehydeadducts inhaled with tobacco smoke, complicated by the fact that all cells of the organism contain endogenous formaldehyde and, accordingly, its adducts. Therefore, while studying the biological effects of exogenous formaldehyde it is necessary to carry out its radioactive labeling.

\section{CONCLUSION}

Analysis of recent data has demonstrated that DNAadducts may be potential markers of LC risk. The appearance of DNA adducts in blood or tissues is the result of the interaction of carcinogenic factors, such as tobacco constituents, and the body reaction which is determined by individual characteristics of metabolicand repair systems. An important fact is that initially most carcinogens of tobacco smoke are inert substances and acquire their genotoxic activity only after metabolic activation. Thus, the ultimate carcinogenic effect of tobacco constituents (initiation of malignancy) manifests as a result of their genotoxic action which can be considerably modified by the functioning of the repair systems. So, DNA adducts may be considered as a cumulative mirror of heterogeneous response of different individuals to smoking carcinogens, which finally could provide the risk for LC.

At the current moment, there are some difficulties impeding the widespread use of DNA adducts as cancer biomarkers. Firstly, there are no analytical standards of substance for quantitative determination of DNA adducts, except for the most common 8-oxo-dG. For the rest of the substances it is necessary to develop commercially available standards, including deuterated forms. Third, in order to detect the increased level of DNA-adducts, it is required to establish background values, because some adducts may be formed endogenous. The level of background DNA damage formed as a result of some endogenous processes may reach 1 to $10^{5}$ according to some reports [67]. Furthermore, the same DNA-adducts can be formed by several pathways, so 8-oxo-dG, may be produced by exposure to benzo[a]pyrene, as well as to carbon tetrachloride $[68,69]$. In our opinion, further studies are needed to establish the laws of the content of individual adducts in tissues and biological fluids of patients, as well as their concentration.

Using of common research method of mass spectrometry as a routine method in clinical laboratory is economically unjustified. Therefore, for the realization of the early detection of LC it is necessary to develop an appropriate method. It is known that all DNA adducts are immunogenic, their accumulation to some threshold concentration leads to the appearance of long-living autoantibodies [70, 71]. Over the past year, increasing attention has focused on the possibility to detect autoantibodies against DNA adducts, in particular adducts of benzo[a]pyrene [72-74]. Thus, identification of autoantibodies to DNA adducts may be a promising approach for early diagnosis of LC in highrisk groups (smokers, manufacturing workers, urban dwellers). The use of high effective multiplex ELISA immunoassay will allow the numerous autoantibodiesagainst different DNA adducts to be detected in order to increase the method sensitivity. 


\section{ACKNOWLEDGEMENT}

This Research was supported by Federal Targeted Programme for Research and Development in Priority Areas of Development of the Russian Scientific and Technological Complex for 2014-2020, "Development of molecular signatures for early detection of lung cancer" (№ 14.575.21.0064 from 05.08.2014).

\section{REFERENCES}

1. GLOBOCAN 2012: Estimated Cancer Incidence, Mortality and Prevalence Worldwide in 2012. International Agency for Research on Cancer (IARC) (http://globocan.iarc.fr).

2. Chissov VI, Starinsky VV. The course of implementation of measures to improve oncological care to the population of Russia. Ros Onkol Zhurnal 2011; 4: 4-8 (in Russian).

3. Mutti A. Molecular diagnosis of lung cancer: an overview of recent development. Acta Biomed 2008; 79: 11-23.

4. Evangelista L, Cervino AR, Ghiotto C, et al. Tumor markerguided PET in breast cancer patients: a recipe for a perfect wedding: a systematic literature review and meta-analysis. Clin Nucl Med 2012; 37: 467-74.

5. Mirabelli P, Incoronato M. Usefulness of traditional serum biomarkers for management of breast cancer patients. BioMed Research Int 2013; Article ID 685641: 9.

6. Molina R, Auge JM, Filella X, et al. Pro-gastrin-releasing peptide (ProGRP) in patients with benign and malignant diseases: comparison with CEA, SCC, CYFRA 21-1 and NSE in patients with lung cancer. Anticancer Res 2005; 25: 1773-8.

7. Rykova EY, Morozkin ES, Ponomaryova AA, et al. Cell-free and cell-bound circulating nucleic acid complexes: mechanisms of generation, concentration and content. Expert Opin Biol Ther 2012; 12: S141-53.

8. Ignatiadis M, Dawson SJ. Circulating tumor cells and circulating tumor DNA for precision medicine: dream or reality? Ann Oncol 2014; 25: 2304-13.

9. Heitzer E, Ulz P, Geigl JB. Circulating tumor DNA as a liquid biopsy for cancer. Clin Chem 2015; 61: 112-23. doi: 10.1373/clinchem.2014.222679.

10. Garaguso I, Halter R, Krzeminski J, et al. Method for the rapid detection and molecular characterization of DNA alkylating agents by MALDI-TOF mass spectrometry. Anal Chem 2010; 82: 8573-82.

11. Hoffmann D, Hecht SS. Advances in tobacco carcinogenesis. In: CS Cooper, PL Grover, eds. Handbook of experimental pharmacology. Heidelberg (Germany): Springer-Verlag, 1990: 63-102.

12. IARC Monographs on evaluation of the carcinogenic risk of chemicals to humans: tobacco smoke and involuntary smoking. Lyon, 2002; 83: 1452.

13. Hecht SS. Tobacco smoke carcinogens and lung cancer. J Natl Cancer Inst 1991; 91: 1194-210.

14. Pfeifer GP, Hainaut P. On the origin of $G \rightarrow T$ transversions in lung cancer. Mutat Res 2003; 526: 39-43.

15. Le Calvez F, Mukeria A, Hunt JD, et al. TP53 and KRAS mutation load and types in lung cancers in relation to tobacco smoke: distinct patterns in never, former, and current smokers. Cancer Res 2005; 65: 5076-83.

16. Hainaut $P$, Pfeifer GP. Patterns of $\mathrm{p} 53 \mathrm{G}->$ T transversions in lung cancers reflect the primary mutagenic signature of DNAdamage by tobacco smoke. Carcinogenesis 2001; 22: 367-74.

17. Hussain SP, Amstad P, Raja K, et al. Mutability of 53 hotspot codons to benzo(a)pyrene diol epoxide (BPDE) and the frequency of $\mathrm{p} 53$ mutations in nontumorous human. Cancer Res 2001; 61: 6350-5.

18. Bowman RV, Yang IA, Semmler AB, et al. Epigenetics of lung. Respirology 2006; 11: 355-65.
19. Belinsky SA, Grimes MJ, Casas E, et al. Predicting gene promoter methylation in non-small cell lung cancer by evaluating sputum and serum. Brit J Cancer 2007; 96: 1278-83.

20. Palmisano WA, Crume KP, Grimes MJ, et al. Aberrant promoter methylation of the transcription factorgenes PAX5 alpha and beta in human cancers. Cancer Res 2003; 63: 4620-5.

21. Ponomaryova AA, Rykova EY, Cherdyntseva NV, et al. RAR 32 gene methylation level in the circulating DNA from blood of patients with lung cancer. Eur J Cancer Prev 2011; 20: 453-5.

22. Ponomaryova AA, Rykova EYu, Cherdyntseva NV, et al. Molecular genetic markers in diagnostic of lung cancer. Mol Biol 2011; 45: 175-89 (in Russian).

23. Valko M, Leibfritz D, Moncol J, et al. Free radicals and antioxidants in normal physiological functions and human disease. Int J Biochem Cell Biol 2007; 39: 44-84.

24. Chekhun VF. Inflammation and cancer. Onkologiya 2009; 11: 244-5 (in Russian).

25. van der Vaart H, Postma DS, Timens W, et al. Acute effects of cigarette smoke on inflammation and oxidative stress: a review. Thorax 2004; 59: 713-21.

26. Hanahan D, Weinberg RA. Hallmarks of cancer: the next generation. Cell 2011; 144: 646-74.

27. Wang XC, Tian LL, Wu HL, et al. Expression of miRNA130a in nonsmall cell lung cancer. Am J Med Sci 2010; 340: 385-8.

28. Gao W, Yu Y, Cao H, et al. Deregulated expression of miR-21, miR-143 and miR-181a in non small cell lung cancer is related to clinicopathologic characteristics or patient prognosis. Biomed Pharmacother 2010; 64: 399-408.

29. Landi MT, Zhao Y, Rotunno M, et al. MicroRNA expression differentiates histology and predicts survival of lung. Clin Cancer Res 2010; 16: 430-41.

30. Imyanitov E.N. Molecular pathology of lung cancer. Pract Oncol 2006; 7: 131-7 (in Russian).

31. Tunali NE, Tiryakioğlu NO. Polymorphisms in the xenobiotic genes and susceptibility to bladder cancer. J Cell Mol Biol 2011; 9: 5-13.

32. Reszka E, Wasowicz W, Gromadzinska J. Genetic polymorphism of xenobiotic metabolising enzymes, diet and cancer susceptibility. Br J Nutr 2006; 96: 609-19.

33. Shimada T. Xenobiotic-metabolizing enzymes involved in activation and detoxification of carcinogenic polycyclic aromatic hydrocarbons. Drug Metabol Pharmacokin 2006; 21: 257-76.

34. Williams JA, Phillips DH. Mammary expression of xenobiotic metabolizing enzymes and their potential role in breast cancer. Cancer Res 2000; 60: 4667-77.

35. Rojas M, Cascorbi I, Alexandrov K, et al. Modulation of benzo[a]pyrene diolepoxide-DNA adduct levels in human white blood cells by CYP1A1, GSTM1 and GSTT1 polymorphism. Carcinogenesis 2000; 21: 35-41.

36. Tang D, Rundle A, Mooney L, et al. Sulfotransferase 1A1 (SULT1A1) polymorphism, PAH-DNA adduct levels in breast tissue and breast cancer risk in a case-control study. Breast Cancer Res Treatment 2003; 78: 217-22.

37. Strickland PT, Kang D, Bowman ED, et al. Identification of 1-hydroxypyrene glucuronide as a major pyrene metabolite in human urine by synchronous fluorescence spectroscopy and gas chromatography-mass spectrometry. Carcinogenesis 1994; 15: 483-7.

38. Obolenskaya MY, Teplyuk NM, Divi, RL, et al. Human placental glutathione S-transferase activity and polycyclic aromatic hydrocarbon DNA adducts as biomarkers for environmental oxidative stress in placentas from pregnant women living in radioactivityand chemically-polluted regions. Toxicol Letters 2010; 196: 80-6.

39. Binkova B, Chvatalova I, Lnenickova Z, et al. PAH-DNA adducts in environmentally exposed population in relation to metabolic and DNA repairgene polymorphisms. Mutat Res/Fund Mol Mech Mutagen 2007; 620: 49-61. 
40. Hung RJ, Boffetta P, Brockmoller J, et al. CYP1A1 and GSTM genetic polymorphisms and lung cancer risk in Caucasian nonsmokers: a pooled analysis. Carcinogenesis 2003; 24: 875-82.

41. Imyanitov EN, Togo AV, Hanson KP. Searching for cancerassociated gene polymorphisms: promises and obstacles. Cancer Lett 2004; 204: 3-14.

42. Shen J, Desai M, Agrawal M, et al. Polymorphisms in nucleotide excision repair genes and DNA repair capacity phenotype in sisters discordant for breast cancer. Cancer Epidemiol Biomark Prev 2006; 15: 1614-9.

43. Monzo M, Moreno I, Navarro A, et al. Single nucleotide polymorphisms in nucleotide excision repair genes XPA, XPD, XPG and ERCC1 in advanced colorectal cancer patients treated with first-line oxaliplatin/fluoropyrimidine. Oncology 2007; 72: 364-70.

44. Meschini R, Berni A, Marotta E, et al. DNA repair mechanisms involved in the removal of DBPDE induced lesions leading to chromosomal alterations in $\mathrm{CHO}$ cells. Cytogen Genome Res 2010; 128: 124-30.

45. Cherdyntseva NV, Sevostjanova NV, Urazova LN, et al. Genetic and epigenetic risk factors for lung cancer. Mol Med 2005; 3: 49-55 (in Russian).

46. Ji G, Gu A, Zhou Y, et al. Interactions between exposure to environmental polycyclic aromatic hydrocarbons and DNA repair gene polymorphisms on bulky DNA adducts in human sperm. PLoS ONE 2010; 5: e13145.

47. Shen M, Hung RJ, Brennan P, et al. Polymorphisms of the DNA repair genes XRCC1, XRCC3, XPD, interaction with environmental exposures, and bladder cancer risk in a case-control study in northern Italy. Cancer Epidemiol Biomark Prev 2003; 12: $1234-40$.

48. Wolterbeek AP, Schoevers EJ, Rutten AA, et al. A critical appraisal of intratracheal instillation of benzo[a]pyrene to Syrian golden hamsters as a model in respiratory tract carcinogenesis. Cancer Lett 1995; 89: 107-16.

49. Huang M, Blair IA, Penning TM. Identification of stable benzo[a]pyrene-7,8-dione-DNA adducts in human lung cells. Chem Res Toxicol 2013; 26: 685-92.

50. Buening MK, Wislocki PG, Levin H, et al. Tumorigenicity of the optical enantiomers of the diastereomeric benzo[a]pyrene 7,8-diol-9,10-epoxides in newborn mice: exceptional activity of (+)-7 $\beta, 8 \alpha$-dihydroxy-9 $\alpha, 10 \alpha$-epoxy-7,8,9,10-tetrahydrobenzo[a] pyrene. Proc Nat Acad Sci USA 1978; 75: 5358-61.

51. Boysen G, Hecht SS. Analysis of DNA and protein adducts of benzo[a]pyrene in human tissues using structure-specific methods. Mut Res/Rev Mut Res 2003; 543: 17-30.

52. Rojas M, Marie B, Vignaud JM, et al. High DNA damage by benzo[a]pyrene 7,8-diol-9,10-epoxide in bronchial epithelial cells from patients with lung cancer: comparison with lung parenchyma. Cancer Lett 2004; 207: 157-63.

53. Lee HW, Wang HT, Weng MW, et al. Acrolein- and 4-Aminobiphenyl-DNA adducts in human bladder mucosa and tumor tissue and their mutagenicity in human urothelial cells. Oncotarget 2014; 5: 3526-40.

54. Arif JM, Dresler C, Clapper ML, et al. Lung DNAadducts detected in human smokers are unrelated to typical polyaromatic carcinogens. Chem Res Toxicol 2006; 19: 295-9.

55. Feng Z, Hu W, Hu Y, et al. Acrolein is a major cigaretterelated lung cancer agent: Preferential binding at p 53 mutational hotspots and inhibition of DNA repair. Proc Natl Acad Sci USA 2006; 103: 15404-9.

56. Nath RG, Ocando JE, Guttenplan JB, et al. 1,N2propanodeoxyguanosine adducts: potential new biomarkers of smoking-inducedDNA damage in human oral tissue. Cancer Res 1998; 58: 581-4.
57. Zhang S, Villalta PW, Wang M, et al. Detection and quantitation of acrolein-derived 1,N2-propanodeoxyguanosine adducts in human lung by liquid chromatography-electrospray ionizationtandem mass. Chem Res Toxicol 2007; 20: 565-71.

58. Wang $M, Y u N$, Chen $L$, et al. Identification of an acetaldehyde adduct in human liver DNA and quantitation as N2-ethyldeoxyguanosine. Chem Res Toxicol 2006; 19: 319-24.

59. Singh R, Kaur B, Farmer PB. Detection of DNA damage derived from a direct acting ethylating agent present in cigarette smoke by use of liquid chromatography-tandem mass spectrometry. Chem Res Toxicol 2005; 18: 249-56.

60. Godschalk R, NairJ, Kliem HC, et al. Modified immunoenriched (32)P-HPLC assay for the detection of O(4)-ethylthymidine in human biomonitoring studies. Chem Res Toxicol 2002; 15:433-7.

61. Hecht SS, Yuan JM, Hatsukami D. Applying tobacco carcinogen and toxicant biomarkers in product regulation and cancer prevention. Chem Res Toxicol 2010; 23: 1001-8.

62. Phillips DH, Venitt S. DNA and protein adducts in human tissues resulting from exposure to tobacco smoke. Int J Cancer 2012; 131: 2733-53.

63. Hecht SS. Biochemistry, biology, and carcinogenicity of tobacco-specific N-nitrosamines. Chem Res Toxicol 1998; 11: $559-603$.

64. Bessette EE, Spivack SD, Goodenough AK. Identification of carcinogen DNA adducts in human saliva by linear quadrupole ion trap/multistage tandem mass spectrometry. Chem Res Toxicol 2010; 23: 1234-44.

65. Wang M, Cheng G, Balbo S, et al. Clear differences in levels of a formaldehyde-DNA adduct in leukocytes of smokers and nonsmokers. Cancer Res 2009; 69: 7170.

66. Lu K, Collins LB, Ru H, et al. Distribution of DNA adducts caused by inhaled formaldehyde is consistent with induction of nasal carcinoma but not leukemia. Toxicol Sci 2010; 116: 441-51.

67. Gupta RC, Lutz WK. Background DNA damage for endogenous and unavoidable exogenous carcinogens: a basis for spontaneous cancer incidence? Mutat Res 1999; 424: 1-8.

68. Marie-Desvergne C, Maitre A, Bouchard M, et al. Evaluation of DNA adducts, DNA and RNA oxidative lesions, and 3-hydroxybenzo(a)pyrene as biomarkers of DNA damage in lung following intravenous injection of the parent compound in rats. Chem Res Toxicol 2010; 23: 1207-14.

69. Tarantini A, Maitre A, Lefebvre E, et al. Relative contribution of DNA strand breaks and DNA adducts to the genotoxicity of benzo[a]pyrene as a pure compound and in complex mixtures. Mutat Res 2009; 671: 67-75.

70. Singh R, Teichert F, Verschoyle RD, et al. Simultaneous determination of 8-oxo-2'-deoxyguanosine and 8-oxo-2'deoxyadenosine in DNA using online column-switching liquid chromatography/tandem mass spectrometry. Rapid Commun Mass Spectrom 2009; 23: 151-60.

71. Petruzzelli S, Celi A, Pulerà N, et al. Serum antibodies to benzo(a)pyrene diol epoxide-DNA adducts in the general population: effects of air pollution, tobacco smoking, and family history of lung diseases. Cancer Res 1998; 58: 4122-6.

72. Pulerà N, Petruzzelli S, Celi A, et al. Presence and persistence of serum anti-benzo[a]pyrene diolepoxide-DNA adduct antibodies in smokers: effects of smoking reduction and cessation. Int J Cancer 1997; 70: 145-9.

73. Ustinov VA, Matveeva VA, Kostyanko MA, et al. Antibodies against benzo[a]pyrene in immunized mouse and in lung cancer patients. Exp Oncol 2013; 35: 207-10.

74. Borska L, Andrys C, Krejsek J, et al. Serum level of antibody against benzo[a]pyrene-7,8-diol-9,10-epoxide-DNA adducts in people dermally exposed to PAHs. J Immunol Res 2014; 2014: 834389 . doi: $10.1155 / 2014 / 834389$. 